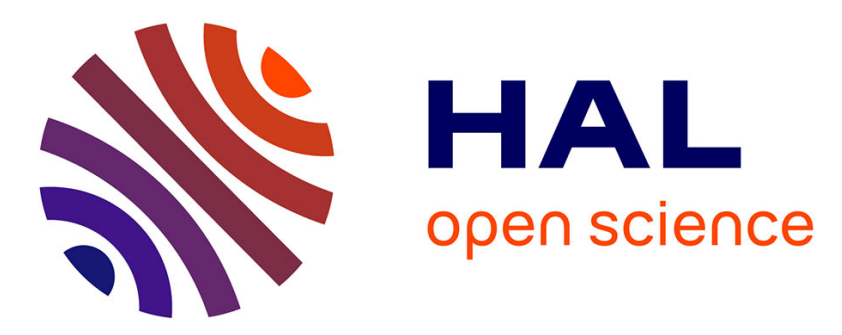

\title{
Homogenization of fluid-porous interface coupling in a biconnected fractured media
}

\author{
Isabelle Gruais, Dan Polisevski
}

\section{To cite this version:}

Isabelle Gruais, Dan Polisevski. Homogenization of fluid-porous interface coupling in a biconnected fractured media. Applicable Analysis, 2015, 94 (8), pp.1736-1747. 10.1080/00036811.2014.952292 . hal-00990929

\section{HAL Id: hal-00990929}

\section{https://hal-univ-rennes1.archives-ouvertes.fr/hal-00990929}

Submitted on 21 May 2014

HAL is a multi-disciplinary open access archive for the deposit and dissemination of scientific research documents, whether they are published or not. The documents may come from teaching and research institutions in France or abroad, or from public or private research centers.
L'archive ouverte pluridisciplinaire HAL, est destinée au dépôt et à la diffusion de documents scientifiques de niveau recherche, publiés ou non, émanant des établissements d'enseignement et de recherche français ou étrangers, des laboratoires publics ou privés. 


\title{
Homogenization of fluid-porous interface coupling in a biconnected fractured media
}

\author{
Isabelle Gruais · Dan Poliševski
}

Received: date / Accepted: date

\begin{abstract}
The modelization of mass transfer through biconnected fractured porous media is studied by homogenizing the coupling between the Darcyan percolation and the viscous Stokes flow on their interface governed by the Beavers-Joseph law. The case of high transmission coefficients is considered. The asymptotic behaviour is completely described with the help of the solutions of some specific local problems and of a non homogeneous Neumann problem defined by the effective permeability tensor.
\end{abstract}

Keywords fractured porous media - Stokes flow · Beavers-Joseph interface • homogenization $\cdot$ two-scale convergence

Mathematics Subject Classification (2010) 35B27 · 76M50 - 76S05

\section{Introduction}

Damaged porosity is the realistic counterpart of the model of porous media that was successfully recovered by means of homogenization methods based on formal asymptotic expansions in [19] and later proved in [20] in its main part, namely the pressure problem. Variants including the influence of cracks have been studied in [3], [8] based on an adequate choice of the physical model, see [3]-[5], [11]-[14], [18] where this issue is taken into account. An important assumption in homogenization theory dealing with porous media is the periodicity of the distribution of inclusions [6], [19], [20], which has given rise to the periodicity-based two-scale convergence method of the pioneering article [16] and numerous improvements by [1], [2], [15].

Isabelle Gruais

Université de Rennes 1, I.R.M.A.R, Campus de Beaulieu, 35042 Rennes Cedex (France)

E-mail: isabelle.gruais@univ-rennes1.fr

Dan Polisevschi

Inst. Math. "S.Stoilow", Romanian Academy, P.O. Box 1-764, Bucharest (Romania)

E-mail: danpolise@yahoo.com 
Darcy's law results from the homogenization theory applied to a periodic distribution of solid blocks immersed in a Stokes fluid. The asymptotic analysis was performed with the help of a restriction operator, which enables a natural extension of the pressure in the blocks. The next step was to consider transmission problems which proved to be a difficult question to handle in asymptotic analysis. In this direction, we have already studied (see [10]) the Stokes problem immersed in a porous medium when the interface is governed by the Beavers-Joseph law. The two-component model is that of a porous medium perforated by cracks filled with Stokes fluid interacting with the ambiant porous medium through Beavers-Joseph conditions, in the case of bounded transfer coefficient. The study is handled by the two-scale convergence method and gives rise to a coupled model between a microscopic Stokes flow and a macroscopic Darcy law.

We consider here an incompressible viscous fluid flow in a periodically structured domain consisting of two interowen regions, separated by an interface. The first region represents the system of fissures which form the connected fracture, where the viscous flow is governed by the Stokes system. The second region, which may be also connected, stands for a porous structure of a certain permeability, where the flow is governed by Darcy's law. These two flows are coupled on the interface by the Saffman's variant [18] of the Beavers-Joseph condition [5], [12] which was confirmed by [11] as the limit of a homogenization process. Besides the continuity of the normal component of the velocity, it imposes the proportionality of the tangential component of the viscous stress. The local problem has vanishing tangential velocity at the interface, which is linking both components of the corresponding microscopic cell problem while being coupled to a macroscopic Darcy law as well. The asymptotic behaviour is completely described with the help of the solutions of the local problems and of a non homogeneous Neumann problem defined by an effective permeability tensor.

The paper is organized as follows. The mathematical problem is presented in Section 2 along with a priori estimates derived from it. The coupling between the porous medium submitted to Darcy's law and the Stokes flow is described in details in Section 3. In particular, the scalings of both components are linked through the modelization of the interface which plays an important part in the asymptotic study. Compactness properties of the sequence of problems are studied in Section 4. The limit problem is introduced in Theorem 7 of Section 5 and described thereafter in Theorem 8. It consists of a macroscopic Darcy system characterized by a permeability tensor having the advantage of being easily computable through a local cell problem.

\section{The properties of the two-connected components structure}

Let $\Omega$ be an open connected bounded set in $\mathbb{R}^{N}(N \geq 2)$, locally located on one side of the boundary $\partial \Omega$, a Lipschitz manifold composed of a finite number of connected components. 
Let $Y_{f}$ be a Lipschitz open connected subset of the unit cube $\left.Y=\right] 0,1\left[{ }^{N}\right.$, such that the intersections of $\partial Y_{f}$ with $\partial Y$ are reproduced identically on the opposite faces of the cube without reaching the edges.

Repeating $Y$ by periodicity, we assume that the reunion of all the $\bar{Y}_{f}$ parts, denoted by $\mathbb{R}_{f}^{N}$, is a connected domain in $\mathbb{R}^{N}$ with a boundary of class $\mathcal{C}^{2}$, such that $\partial Y_{f}$ is Lipschitz-continuous. Defining $Y_{s}=Y \backslash \bar{Y}_{f}$, we assume also that the reunion of all the $\bar{Y}_{s}$ parts is a connected domain in $\mathbb{R}^{N}$. We set the origin of the coordinates system in such a point that there exists $\delta>0$ for which $B(0, \delta) \subset \mathbb{R}_{s}^{N}=\mathbb{R}^{N} \backslash \overline{\mathbb{R}}_{f}^{N}$. The normal on $\Gamma=\bar{Y}_{f} \cap \bar{Y}_{s}$, outward with respect to $Y_{f}$, is denoted by $\nu$.

For any $\varepsilon \in] 0,1[$ we denote

$$
\begin{gathered}
Z_{\varepsilon}=\left\{k \in \mathbb{Z}^{N}, \varepsilon k+\varepsilon Y \subseteq \Omega\right\} \\
I_{\varepsilon}=\left\{k \in Z_{\varepsilon}, \varepsilon k \pm \varepsilon e_{i}+\varepsilon Y \subseteq \Omega, \forall i \in \overline{1, N}\right\}
\end{gathered}
$$

where $e_{i}$ are the unit vectors of the canonical basis in $\mathbb{R}^{N}$.

Finally, we define the system of fissures by

$$
\Omega_{\varepsilon f}=\operatorname{int}\left(\cup_{k \in I_{\varepsilon}}\left(\varepsilon k+\varepsilon \bar{Y}_{f}\right)\right)
$$

and the porous matrix of our structure by $\Omega_{\varepsilon s}=\Omega \backslash \bar{\Omega}_{\varepsilon f}$. The interface between the porous blocks and the fluid is denoted by $\Gamma_{\varepsilon}=\partial \Omega_{\varepsilon f}$.

Obviously, $\Omega_{\varepsilon s}$ and $\Omega_{\varepsilon f}$ are connected and the fracture ratios of this structure have the property: $\left.\left|\Omega_{\varepsilon f}\right| /|\Omega| \rightarrow m:=\left|Y_{f}\right| \in\right] 0,1[$, when $\varepsilon \rightarrow 0$.

A useful property of the present structure is the existence of a bounded extension operator (see [17]) similar to that introduced in [6] (see also [7]) in the case of isolated fractures.

Theorem 1 There exists an extension operator $P_{\varepsilon}: H^{1}\left(\Omega_{\varepsilon f}\right) \rightarrow H^{1}(\Omega)$ such that

$$
\begin{gathered}
P_{\varepsilon} u=u \quad \text { in } \Omega_{\varepsilon f} \\
\left|e\left(P_{\varepsilon} u\right)\right|_{L^{2}(\Omega)} \leq C|e(u)|_{L^{2}\left(\Omega_{\varepsilon f}\right)}, \quad \forall u \in H^{1}\left(\Omega_{\varepsilon f}\right)
\end{gathered}
$$

where $C$ is independent of $\varepsilon$.

For any $h \in\{s, f\}$, we recall the main inequalities that hold here.

Lemma 1 There exists $C>0$ independent of $\varepsilon$ such that

$$
\begin{gathered}
|u|_{L^{2}\left(\Omega_{\varepsilon f}\right)} \leq C \varepsilon|\nabla u|_{L^{2}\left(\Omega_{\varepsilon f}\right)}, \quad \forall u \in H_{0}^{1}\left(\Omega_{\varepsilon f}\right) \\
|u|_{L^{2}\left(\Omega_{\varepsilon s}\right)} \leq C\left(\varepsilon|\nabla u|_{L^{2}\left(\Omega_{\varepsilon s}\right)}+\varepsilon^{1 / 2}|u|_{L^{2}\left(\partial \Omega_{\varepsilon s}\right)}\right), \quad \forall u \in H^{1}\left(\Omega_{\varepsilon s}\right)
\end{gathered}
$$

Theorem 2 There exists $C>0$ independent of $\varepsilon$ such that

$$
|p|_{L^{2}\left(\Omega_{\varepsilon h}\right) / \mathbb{R}} \leq \frac{C}{\varepsilon}|\nabla p|_{H^{-1}\left(\Omega_{\varepsilon h}\right)}, \quad \forall p \in L^{2}\left(\Omega_{\varepsilon h}\right) .
$$


Corollary 1 There exists $C>0$ independent of $\varepsilon$ such that

$$
|p|_{L^{2}\left(\Omega_{\varepsilon h}\right) / \mathbb{R}} \leq C|\nabla p|_{L^{2}\left(\Omega_{\varepsilon h}\right)}, \quad \forall p \in H^{1}\left(\Omega_{\varepsilon h}\right) .
$$

Denoting $\chi_{\varepsilon h}(x)=\chi_{h}\left(\frac{x}{\varepsilon}\right)$, where $\chi_{h}$ is the characteristic function of $Y_{h}$ in $Y, h \in\{s, f\}$, we have a specific compactness result (see [17]) for the pressure type estimates in $H^{-1}\left(\Omega_{\varepsilon f}\right)$.

Theorem 3 For every $\varepsilon \in] 0,1\left[\right.$, let $q^{\varepsilon} \in L^{2}\left(\Omega_{\varepsilon f}\right)$, such that for some constant $C>0$ there hold:

$$
\begin{aligned}
& \left.\left|\int_{\Omega_{\varepsilon f}} q^{\varepsilon}(x) d x\right| \leq C, \quad \forall \varepsilon \in\right] 0,1[. \\
& \left.\left|\nabla q^{\varepsilon}\right|_{H^{-1}\left(\Omega_{\varepsilon f}\right)} \leq C \varepsilon, \quad \forall \varepsilon \in\right] 0,1[.
\end{aligned}
$$

Then, there exists $q \in L^{2}(\Omega)$ such that, on some subsequence:

$$
\chi_{\varepsilon f} q^{\varepsilon} \stackrel{2}{\rightarrow} \chi_{f} q .
$$

\section{The coupled Darcy-Stokes percolation}

A model of fluid flow through a fractured porous medium is associated to our structure by assuming that there is a filtration flow in $\Omega_{\varepsilon s}$ obeying the Darcy's law and that there is a viscous flow in $\Omega_{\varepsilon f}$ governed by the Stokes system. These two flows are coupled by a Saffman's variant [18] of the BeaversJoseph condition [5], [12]. The system is completed by an impermeability condition on $\partial \Omega$ :

$$
\begin{gathered}
\operatorname{div} u^{\varepsilon}=0 \quad \text { in } \Omega \\
A^{\varepsilon} u^{\varepsilon}=g-\nabla p^{\varepsilon s} \quad \text { in } \quad \Omega_{\varepsilon s}, \quad g \in L^{2}(\Omega) \\
\sigma_{i j}^{\varepsilon f}=-p^{\varepsilon f} \delta_{i j}+\varepsilon^{2} e_{i j}\left(u^{\varepsilon}\right) \quad \text { in } \Omega_{\varepsilon f} \\
-\frac{\partial}{\partial x_{j}} \sigma_{i j}^{\varepsilon f}=g_{i} \quad \text { in } \Omega_{\varepsilon f} \\
v^{\varepsilon s} \cdot \nu^{\varepsilon}=v^{\varepsilon f} \cdot \nu^{\varepsilon} \quad \text { on } \Gamma_{\varepsilon}, \\
p^{\varepsilon s} \nu_{i}^{\varepsilon}+\sigma_{i j}^{\varepsilon f} \nu_{j}^{\varepsilon}+\varepsilon^{r} \alpha_{\varepsilon}\left(u_{i}^{\varepsilon f}-\left(u^{\varepsilon f} \cdot \nu^{\varepsilon}\right) \nu_{i}^{\varepsilon}\right)=0 \quad \text { on } \Gamma_{\varepsilon}, \quad r<1 \\
u^{\varepsilon} \cdot n=0 \quad \text { on } \partial \Omega, \quad n \text { the outward normal on } \partial \Omega,
\end{gathered}
$$

where $u^{\varepsilon s}, u^{\varepsilon f}$ and $p^{\varepsilon s}, p^{\varepsilon f}$ stand for the corresponding velocities and pressures,

$$
\begin{gathered}
A^{\varepsilon}(x)=A\left(\frac{x}{\varepsilon}\right), \quad A \in\left(\mathcal{C}_{\text {per }}^{\infty}(Y)\right)^{N \times N} \quad \text { coercive, symmetric } \\
\alpha^{\varepsilon}(x)=\alpha\left(\frac{x}{\varepsilon}\right) \geq \alpha_{0}>0, \quad \alpha \in \mathcal{C}_{\text {per }}^{1}(\bar{Y})
\end{gathered}
$$


and $e(v)$ denotes the symmetric tensor of the velocity gradient defined by

$$
e_{i j}(v)=\frac{1}{2}\left(\frac{\partial v_{i}}{\partial x_{j}}+\frac{\partial v_{j}}{\partial x_{i}}\right) .
$$

As usual, we use the notations:

$$
\begin{gathered}
H_{0}(\operatorname{div}, \Omega)=\{v \in H(\operatorname{div}, \Omega), \quad v \cdot n=0 \quad \text { on } \quad \partial \Omega\} \\
L_{0}^{2}(\Omega)=\left\{p \in L^{2}(\Omega), \quad \int_{\Omega} p=0\right\} \\
V_{0}(\operatorname{div}, \Omega)=\left\{v \in H_{0}(\operatorname{div}, \Omega), \quad \operatorname{div} v=0 \quad \text { in } \quad \Omega\right\}
\end{gathered}
$$

Next, we define

$$
H_{\varepsilon}=\left\{v \in H_{0}(\operatorname{div}, \Omega), \quad v \in H^{1}\left(\Omega_{\varepsilon f}\right)^{N}\right\},
$$

the Hilbert space endowed with the scalar product

$(u, v)_{H_{\varepsilon}}=\int_{\Omega_{\varepsilon s}} u \cdot v+\int_{\Omega_{\varepsilon s}} \operatorname{div} u \operatorname{div} v+\varepsilon^{2} \int_{\Omega_{\varepsilon f}} e(u) e(v)+\varepsilon^{r} \int_{\Gamma_{\varepsilon}}\left(\gamma^{\varepsilon} u-\left(\gamma_{\nu}^{\varepsilon} u\right) \nu^{\varepsilon}\right) \gamma^{\varepsilon} v$,

where $\gamma^{\varepsilon}$ and $\gamma_{\nu}^{\varepsilon}$ denote respectively the trace and the normal trace operators on $\Gamma_{\varepsilon}$, while $\nu^{\varepsilon}$ is the normal on $\Gamma_{\varepsilon}$, outward with respect to $\Omega_{\varepsilon f}$. The corresponding subspace of incompressible velocities is

$$
V_{\varepsilon}=\left\{v \in V_{0}(\operatorname{div}, \Omega), \quad v \in H^{1}\left(\Omega_{\varepsilon f}\right)^{N}\right\} .
$$

Via the corresponding Korn inequality, we obtain a specific inequality:

Lemma 2 There exists some constant $C>0$, independent of $\varepsilon$, such that

$$
|u|_{L^{2}\left(\Omega_{\varepsilon f}\right)}+\varepsilon|\nabla u|_{L^{2}\left(\Omega_{\varepsilon f}\right)} \leq C|u|_{H_{\varepsilon}}, \quad \forall u \in H_{\varepsilon} .
$$

Looking for the variational formulation of the problem (3.1)-(3.7), we define for any $u, v \in H_{\varepsilon}$ and $q \in L_{0}^{2}(\Omega)$

$$
\begin{gathered}
a_{\varepsilon}(u, v):=\int_{\Omega_{\varepsilon s}} A^{\varepsilon} u \cdot v+\varepsilon^{2} \int_{\Omega_{\varepsilon f}} e(u): e(v)+\varepsilon^{r} \int_{\Gamma_{\varepsilon}} \alpha^{\varepsilon}\left(\gamma^{\varepsilon} u-\left(\gamma_{\nu}^{\varepsilon} u\right) \nu^{\varepsilon}\right) \cdot \gamma^{\varepsilon} v \\
b_{\varepsilon}(q, v):=-\int_{\Omega} q \operatorname{div} v .
\end{gathered}
$$

We see that if the pair $\left(u^{\varepsilon}, p^{\varepsilon}\right)$ is a smooth solution of the problem (3.1)-(3.7), then it is also a solution of the following problem:

To find $\left(u^{\varepsilon}, p^{\varepsilon}\right) \in H_{\varepsilon} \times L_{0}^{2}(\Omega)$ such that

$$
\begin{gathered}
a_{\varepsilon}\left(u^{\varepsilon}, v\right)+b_{\varepsilon}\left(p^{\varepsilon}, v\right)=\int_{\Omega} g^{\varepsilon} \cdot v, \quad \forall v \in H_{\varepsilon}, \\
b_{\varepsilon}\left(q, u^{\varepsilon}\right)=0, \quad \forall q \in L_{0}^{2}(\Omega) .
\end{gathered}
$$


Theorem 4 There exists a unique pair $\left(u^{\varepsilon}, p^{\varepsilon}\right) \in H_{\varepsilon} \times L_{0}^{2}(\Omega)$ solution of (3.19)-(3.20).

Proof. As $H_{0}^{1}(\Omega)$ is obviously included in $H_{\varepsilon}$, the following inf-sup condition is easily satisfied by $b_{\varepsilon}$ :

$$
\exists C_{1}^{\varepsilon}>0 \quad \text { such that } \inf _{q \in L_{0}^{2}(\Omega)} \sup _{v \in H_{\varepsilon}} \frac{b_{\varepsilon}(q, v)}{|v|_{H_{\varepsilon}}|q|_{L_{0}^{2}(\Omega)}} \geq C_{1}^{\varepsilon} .
$$

The positivity conditions (3.8) and (3.9) imply that

$$
\exists C_{2}^{\varepsilon}>0 \quad \text { such that } a_{\varepsilon}(v, v) \geq C_{2}^{\varepsilon}|v|_{H_{\varepsilon}}^{2}, \quad \forall v \in H_{\varepsilon},
$$

that is the $V_{\varepsilon}$-ellipticity of $a_{\varepsilon}$. As we also have

$$
V_{\varepsilon}=\left\{v \in H_{\varepsilon}, \quad b_{\varepsilon}(q, v)=0, \quad \forall q \in L_{0}^{2}(\Omega)\right\},
$$

the proof is completed by Corollary 4.1, Ch. 1 of [9].

\section{A priori estimates and two-scale convergences}

From now on, for any function $\varphi$ defined on $\Omega \times Y$ we shall use the notations

$$
\begin{aligned}
\varphi^{h} & =\left.\varphi\right|_{\Omega \times Y_{h}}, \quad \tilde{\varphi}^{h}=\frac{1}{\left|Y_{h}\right|} \int_{Y_{h}} \varphi(\cdot, y) d y, \quad h \in\{s, f\}, \\
\tilde{\varphi} & =\int_{Y} \varphi(\cdot, y) d y, \quad \text { that is } \quad \tilde{\varphi}=(1-m) \tilde{\varphi}^{s}+m \tilde{\varphi}^{f} .
\end{aligned}
$$

Also, for any sequence $\left(\varphi^{\varepsilon}\right)_{\varepsilon}$, bounded in $L^{2}(\Omega \times Y)$, we denote

$$
\varphi^{\varepsilon} \stackrel{2}{\rightarrow} \varphi
$$

iff $\varphi^{\varepsilon}$ is two-scale convergent to $\varphi \in L^{2}(\Omega \times Y)$ in the sense of [2].

As usual, the asymptotic study starts by the search of a priori estimates of the velocity. Noticing that $u^{\varepsilon} \in V_{\varepsilon}$ and setting $v=u^{\varepsilon}$ in (3.19) we get

$$
\left|u^{\varepsilon}\right|_{H_{\varepsilon}}^{2} \leq C\left|u^{\varepsilon}\right|_{L^{2}(\Omega)}
$$

and therefore:

$$
\left|u^{\varepsilon}\right|_{L^{2}\left(\Omega_{\varepsilon s}\right)} \leq C, \quad \varepsilon\left|e\left(u^{\varepsilon}\right)\right|_{L^{2}\left(\Omega_{\varepsilon f}\right)} \leq C, \quad \varepsilon^{r / 2}\left|\gamma_{\tau}^{\varepsilon} u^{\varepsilon}\right|_{L^{2}\left(\Gamma_{\varepsilon}\right)} \leq C
$$

where, for any $v \in H^{1}\left(\Omega_{\varepsilon f}\right)$, we define $\gamma_{\tau}^{\varepsilon} v \in H^{1 / 2}\left(\Gamma_{\varepsilon}\right)$ by

$$
\gamma_{\tau}^{\varepsilon} v:=\gamma^{\varepsilon} v-\left(\gamma_{\nu}^{\varepsilon} v\right) \nu^{\varepsilon}
$$

We find that $\left(\chi_{\varepsilon s} u^{\varepsilon}\right)_{\varepsilon},\left(\chi_{\varepsilon f} u^{\varepsilon}\right)_{\varepsilon}$ and $\left(\varepsilon \chi_{\varepsilon f} \frac{\partial u^{\varepsilon}}{\partial x_{i}}\right)_{\varepsilon}$ are bounded in $\left(L^{2}(\Omega)\right)^{N}$, $\forall i \in\{1,2, \cdots, N\}$. Using the compacity results of $[16], \exists u \in L^{2}(\Omega \times Y)^{N}$ such that, on some subsequence

$$
u^{\varepsilon} \stackrel{2}{\rightarrow} u
$$




$$
\begin{gathered}
\varepsilon \chi_{\varepsilon f} \nabla u_{i}^{\varepsilon} \stackrel{2}{\rightarrow} \nabla_{y} u_{i} \\
u^{\varepsilon} \rightarrow \tilde{u} \in V_{0}(\operatorname{div}, \Omega) \quad \text { weakly in } L^{2}(\Omega)^{N} .
\end{gathered}
$$

Moreover, denoting

$$
\begin{aligned}
H_{\text {per }}(\operatorname{div}, Y) & =\left\{\varphi \in H_{\text {loc }}\left(\operatorname{div}, \mathbb{R}^{N}\right), \quad \varphi \quad \text { is } Y \text {-periodic }\right\} \\
H_{\text {per }}^{1}\left(Y_{f}\right) & =\left\{\varphi \in H_{\text {loc }}^{1}\left(\mathbb{R}_{f}^{N}\right), \quad \varphi \quad \text { is } Y \text {-periodic }\right\}
\end{aligned}
$$

we can sum the convergence results obtained until now by

Theorem 5 There exists $u \in L^{2}\left(\Omega, H_{\mathrm{per}}(\operatorname{div}, Y)\right)$ with the properties:

$$
\begin{gathered}
u^{f} \in L^{2}\left(\Omega, H_{\text {per }}^{1}\left(Y_{f}\right)^{N}\right), \quad \tilde{u} \in H_{0}(\operatorname{div}, \Omega), \\
\operatorname{div}_{y} u=0 \quad \text { in } \quad \Omega \times Y, \\
\operatorname{div} \tilde{u}=0 \quad \text { in } \quad \Omega,
\end{gathered}
$$

such that the convergences (4.6) and (4.7) hold on some subsequence.

Next, we look for a priori estimates of the pressure. For this, set $v \in H_{\varepsilon}$ in (3.19)-(3.20) such that

$$
v \in H_{0}^{1}(\Omega)^{N} \text { and } v=0 \text { in } \Omega_{\varepsilon f} .
$$

Using the classical $L^{2}$ decomposition, we find that $\exists p^{\varepsilon s} \in H^{1}\left(\Omega_{\varepsilon s}\right)$ such that

$$
\nabla p^{\varepsilon s}=A^{\varepsilon} u^{\varepsilon}-g^{\varepsilon} \in L^{2}\left(\Omega_{\varepsilon s}\right)
$$

and recalling (4.4), we find that there exists $C>0$, independent of $\varepsilon$, such that

$$
\left|\nabla p^{\varepsilon s}\right|_{L^{2}\left(\Omega_{\varepsilon s}\right)} \leq C .
$$

Consequently, using Corollary 1 we have also

$$
\left|p^{\varepsilon s}-\tilde{p}^{\varepsilon s}\right|_{L^{2}\left(\Omega_{\varepsilon s}\right)} \leq C, \quad C>0 \text { independent of } \varepsilon .
$$

It remains to estimate $\tilde{p}^{\varepsilon s}$, and implicitly $\tilde{p}^{\varepsilon f}$, as $\tilde{p}^{\varepsilon}=0$. We remark here that for any $v \in H_{\varepsilon}$ we get

$$
\begin{aligned}
& \frac{|\Omega|}{\left|\Omega_{\varepsilon f}\right|} \tilde{p}^{\varepsilon s} \int_{\Gamma_{\varepsilon}} \gamma_{\nu}^{\varepsilon} v=\int_{\Omega_{\varepsilon s}}\left(p^{\varepsilon s}-\tilde{p}^{\varepsilon s}\right) \operatorname{div} v+\int_{\Omega_{\varepsilon f}}\left(p^{\varepsilon f}-\tilde{p}^{\varepsilon f}\right) \operatorname{div} v-\int_{\Omega} g v+ \\
& \quad+\int_{\Omega_{\varepsilon s}} A^{\varepsilon} u^{\varepsilon} v+\varepsilon^{2} \int_{\Omega_{\varepsilon f}} e\left(u^{\varepsilon}\right): e(v)+\varepsilon^{r} \int_{\Gamma_{\varepsilon}} \alpha^{\varepsilon} u^{\varepsilon}\left(v-\left(\gamma_{\nu}^{\varepsilon} v\right) \nu^{\varepsilon}\right) .
\end{aligned}
$$

In oder to conclude, we need a proper test function. For this, let $\theta \in H_{0}^{1}(Y)^{N}$ such that $\theta=\nu$ on $\Gamma$. Next, we define $\theta^{\varepsilon} \in H_{0}^{1}(\Omega)^{N}$ by

$$
\theta^{\varepsilon}(x)= \begin{cases}\theta\left(\frac{x}{\varepsilon}\right) & \text { for } x \in \Omega_{\varepsilon}=\operatorname{int}\left(\cup_{k \in I_{\varepsilon}}(\varepsilon k+\varepsilon \bar{Y})\right) \\ 0 & \text { for } x \in \Omega \backslash \bar{\Omega}_{\varepsilon} ;\end{cases}
$$


Setting $v=\varepsilon \theta^{\varepsilon}$ in (4.18), we finally get

$$
\left|\tilde{p}^{\varepsilon s}\right| \leq C\left|\Gamma_{\varepsilon}\right|^{-1}\left(1+\left|\theta^{\varepsilon}\right|_{H^{1}(\Omega)}\right),
$$

that is,

$$
\left(\tilde{p}^{\varepsilon s}\right)_{\varepsilon} \quad \text { and } \quad\left(\tilde{p}^{\varepsilon f}\right)_{\varepsilon} \quad \text { are bounded. }
$$

Consequently, there exists $C>0$ independent of $\varepsilon$, such that

$$
\left|p^{\varepsilon s}\right|_{H^{1}\left(\Omega_{\varepsilon s}\right)} \leq C
$$

and using the compactness results of [2], there exists $p^{s} \in H^{1}(\Omega)$ such that

$$
\chi_{\varepsilon s} p^{\varepsilon} \stackrel{2}{\rightarrow} \chi_{s} p^{s}, \quad \text { on some subsequence. }
$$

Next, we set $\psi \in H_{\varepsilon}$ in (3.19)-(3.20) such that $\psi=0$ in $\Omega_{\varepsilon s}$. It follows that $\exists p^{\varepsilon f} \in L^{2}\left(\Omega_{\varepsilon f}\right)$ such that

$$
\left\langle\nabla p^{\varepsilon f}, \psi\right\rangle_{H^{-1}\left(\Omega_{\varepsilon f}\right)}=\varepsilon^{2} \int_{\Omega_{\varepsilon f}} e\left(u^{\varepsilon}\right) e(\psi)-\int_{\Omega_{\varepsilon f}} g^{\varepsilon} \psi, \quad \forall \psi \in H_{0}^{1}\left(\Omega_{\varepsilon f}\right)^{N} .
$$

Consequently, recalling (4.4) and (4.21) we find that $\exists C>0$, independent of $\varepsilon$, such that

$$
\left|\nabla p^{\varepsilon f}\right|_{H^{-1}\left(\Omega_{\varepsilon f}\right)} \leq C \varepsilon .
$$

In the light of (4.21), (4.25) and applying Theorem 3 we find that there exists $p^{f} \in L^{2}(\Omega)$ such that

$$
\chi_{\varepsilon f} p^{\varepsilon} \stackrel{2}{\rightarrow} \chi_{f} p^{f}, \quad \text { on some subsequence. }
$$

Lemma 3 The limits in (4.23) and (4.26) satisfy:

$$
p^{f}=p^{s} \quad \text { in } \quad L^{2}(\Omega) .
$$

Proof. Let $\varphi \in \mathcal{D}(\Omega), \psi \in \mathcal{C}_{\text {per }}^{\infty}(Y)^{N}$ such that

Set

$$
\psi=\left(\gamma_{\nu} \psi\right) \nu \quad \text { on } \quad \Gamma \text { and } \quad \int_{\Gamma} \gamma_{\nu} \psi \neq 0 .
$$

$$
v_{i}^{\varepsilon}(x)=\varepsilon \varphi(x) \psi_{i}\left(\frac{x}{\varepsilon}\right), \quad \forall i \in\{1, \cdots, N\},
$$

in the variational formulation (3.19)-(3.20). Passing to the limit as $\varepsilon \rightarrow 0$, we obtain

$$
\int_{\Gamma} \gamma_{\nu} \psi d \sigma \int_{\Omega}\left(p^{f}(x)-p^{s}(x)\right) \varphi(x) d x=0, \forall \varphi \in \mathcal{D}(\Omega),
$$

which concludes the proof.

The convergence results concerning the pressure can be summed up by

Theorem 6 There exists $p \in \tilde{H}^{1}(\Omega):=L_{0}^{2}(\Omega) \cap H^{1}(\Omega)$ such that on some subsequence we have

$$
p^{\varepsilon} \stackrel{2}{\rightarrow} p
$$


A characteristic property issued from the Beavers-Joseph transfer condition is given by

Lemma 4 The limit u satisfies:

$$
\gamma u^{f}=\left(\gamma_{\nu} u^{f}\right) \nu \quad \text { on } \quad \Omega \times \Gamma .
$$

Proof. For $\varphi \in \mathcal{D}\left(\Omega, H_{\text {per }}^{1}\left(Y_{f}\right)^{N}\right)$ we define

$$
I_{\varepsilon}(\varphi)=\varepsilon \int_{\Gamma_{\varepsilon}} \alpha^{\varepsilon} \gamma^{\varepsilon} u^{\varepsilon}\left(\gamma^{\varepsilon} \varphi-\left(\gamma_{\nu}^{\varepsilon} \varphi\right) \nu^{\varepsilon}\right) .
$$

As $\alpha$ and $\Gamma$ are assumed smooth enough, there exists $\Phi \in \mathcal{C}^{\infty}\left(\Omega, C_{\mathrm{per}}^{1}\left(Y_{f}\right)\right)^{N \times N}$ such that

$$
\Phi_{i j}(x, y)=\alpha(y)\left(\varphi_{i}(x, y)-(\varphi(x, y) \cdot \nu(y)) \nu_{i}(y)\right) \nu_{j}(y) \quad \text { on } \quad \Omega \times \Gamma .
$$

Using the notation

$$
\Phi^{\varepsilon}(x)=\Phi\left(x, \frac{x}{\varepsilon}\right)
$$

we find that

$$
\begin{gathered}
I_{\varepsilon}(\varphi)=\varepsilon \int_{\partial \Omega_{\varepsilon f}} \Phi_{i j}^{\varepsilon} u_{i}^{\varepsilon} \nu_{j}^{\varepsilon} d \sigma= \\
=\int_{\Omega_{\varepsilon f}}\left(\frac{\partial \Phi_{i j}^{\varepsilon}}{\partial y_{j}}\right)^{\varepsilon} u_{i}^{\varepsilon}+\varepsilon \int_{\Omega_{\varepsilon f}}\left(\frac{\partial \Phi_{i j}}{\partial x_{j}}\right)^{\varepsilon} u_{i}^{\varepsilon}+\varepsilon \int_{\Omega_{\varepsilon f}} \Phi_{i j}^{\varepsilon} \frac{\partial u_{i}^{\varepsilon}}{\partial x_{j}}
\end{gathered}
$$

and a direct computation yields

$$
\begin{gathered}
\lim _{\varepsilon \rightarrow 0} I_{\varepsilon}(\varphi)=\int_{\Omega \times Y_{f}}\left(\frac{\partial \Phi_{i j}}{\partial y_{j}} u_{i}^{f}+\Phi_{i j} \frac{\partial u_{i}^{f}}{\partial y_{j}}\right)=\int_{\Omega \times Y_{f}} \Phi_{i j} u_{i}^{f} \nu_{j}= \\
=\int_{\Omega \times \Gamma} \alpha \gamma u^{f}\left(\gamma \varphi-\left(\gamma_{\nu} \varphi\right) \nu\right) .
\end{gathered}
$$

Setting $v=\varepsilon^{1-r} \varphi$ as test function in (3.19) and passing to the limit with $\varepsilon \rightarrow 0$ we find that

$$
\lim _{\varepsilon \rightarrow 0} I_{\varepsilon}(\varphi)=0
$$

that is,

$$
\left.\int_{\Omega \times \Gamma} \alpha \gamma u^{f}\left(\gamma \varphi-\left(\gamma_{\nu} \varphi\right) \nu\right)\right)=0, \quad \forall \varphi \in \mathcal{D}\left(\Omega, H_{\mathrm{per}}^{1}\left(Y_{f}\right)^{N}\right),
$$

which yields the result. 


\section{The two-scale homogenized problem}

Here we find the so-called two-scale homogenized problem, verified by the limits $u$ and $p$, given by Theorems 5 and 6 . By proving that this problem is well-posed, it turns out that all the convergences in Theorems 5,6 and so forth hold on the entire sequence. We conclude that the asymptotic behaviour of $\left(u^{\varepsilon}, p^{\varepsilon}\right)$ is completely described by $(u, p)$, the unique solution of the homogenized problem.

Denoting

$$
\gamma_{\tau} v=\gamma v-\left(\gamma_{\nu} v\right) \nu, \quad \forall v \in H^{1}\left(Y_{f}\right),
$$

we introduce the Hilbert space

$$
\begin{array}{r}
X=\left\{v \in L ^ { 2 } \left(\Omega, H_{\mathrm{per}}(\operatorname{div}, Y), v^{f} \in L^{2}\left(\Omega, H_{\mathrm{per}}^{1}\left(Y_{f}\right)^{N}\right), \tilde{v} \in H_{0}(\operatorname{div}, \Omega),\right.\right. \\
\left.\operatorname{div}_{y} v=0 \quad \text { in } \Omega \times Y, \quad \gamma_{\tau} v^{f}=0 \quad \text { on } \quad \Omega \times \Gamma\right\},
\end{array}
$$

endowed with the scalar product

$$
(u, \varphi)_{X}=\int_{\Omega \times Y_{s}} u \cdot \varphi+\int_{\Omega} \operatorname{div} \tilde{u} \operatorname{div} \tilde{\varphi}+\int_{\Omega \times Y_{f}} e_{y}(u): e_{y}(\varphi) .
$$

Introducing

$$
\begin{gathered}
X_{0}=\{v \in X, \quad \operatorname{div} \tilde{v}=0 \quad \text { in } \quad \Omega\}, \\
M=\tilde{H}^{1}(\Omega) . \\
b(q, v)=-\int_{\Omega} q \operatorname{div} \tilde{v}, \quad \forall(q, v) \in M \times X,
\end{gathered}
$$

we consider the problem:

To find $(v, q) \in X \times M$ such that

$$
\begin{gathered}
a(v, \varphi)+b(q, \varphi)=\int_{\Omega} g \cdot \tilde{\varphi}, \quad \forall \varphi \in X \\
b(\pi, v)=0, \quad \forall \pi \in M
\end{gathered}
$$

where $b$ is given by (5.6) and $a$ is defined by

$$
a(v, \varphi)=\int_{\Omega \times Y_{s}} A v \cdot \varphi+\int_{\Omega \times Y_{f}} e_{y}(v): e_{y}(\varphi) .
$$

Lemma 5 The problem (5.7)-(5.8) is well-defined.

Proof. First, we notice that

$$
X_{0}=\{v \in X, \quad b(q, v)=0, \quad \forall q \in M\} .
$$

As the $X_{0}$-ellipticity of $a$ in $X$ is obvious, the proof is completed by Corollary 4.1, Ch.1 of [9], the corresponding inf-sup condition being justified via the surjectivity of $\operatorname{div} \in \mathcal{L}\left(H_{0}^{1}(\Omega)^{N}, L_{0}^{2}(\Omega)\right)$.

The fact that (5.7)-(5.8) is the homogenized problem of the present case is justified below. 
Theorem $7 u$ and $p$ introduced by Theorems 5 and 6 form a pair $(u, p) \in$ $X \times M$ which is the unique solution of the problem (5.7)-(5.8).

Proof. Let $\varphi \in X \cap \mathcal{D}\left(\Omega, C_{\text {per }}^{\infty}(Y)\right)^{N}$. Denoting, as usual, $\varphi^{\varepsilon}(x)=\varphi\left(x, \frac{x}{\varepsilon}\right)$, we can set $v(x)=\varphi^{\varepsilon}(x)$ in (3.19). Passing to the limit with $\varepsilon \rightarrow 0$, taking advantage of the property $\gamma_{\tau}^{\varepsilon} \varphi^{\varepsilon}=0$ by construction and using the two-scale convergences of Theorems 5 and 6 , we obtain:

and

$$
b_{\varepsilon}\left(p^{\varepsilon}, \varphi^{\varepsilon}\right) \rightarrow-\int_{\Omega \times Y_{s}} p\left(\operatorname{div}_{x} \varphi\right)=b(p, \varphi),
$$

$$
a_{\varepsilon}\left(u^{\varepsilon}, \varphi^{\varepsilon}\right) \rightarrow a(u, \varphi),
$$

which concludes the proof.

The solution of the homogenized problem (5.7)-(5.8) has a further description which takes into account the so-called local problems and effective coefficients. For this, let us introduce the unique solution of the Darcy-Stokes system in $Y$, with the classical normal flow coupling conditions:

For every $i \in\{1, \cdots, N\}, w^{i} \in W$ is the solution of the problem:

$$
\int_{Y_{s}} A w^{i} \psi+\int_{Y_{f}} e_{y}\left(w^{i}\right) e_{y}(\psi)=\int_{Y} \psi_{i}, \quad \forall \psi \in W
$$

where

$W=\left\{w \in H_{\text {per }}(\operatorname{div}, Y), w^{f} \in H_{\text {per }}^{1}\left(Y_{f}\right)^{N}, \operatorname{div} w=0\right.$ in $Y, \gamma_{\tau} w^{f}=0$ on $\left.\Gamma\right\}$.

Next, let $q \in \tilde{H}^{1}(\Omega)$ be the unique solution of the following compatible nonhomogeneous Neumann problem:

$$
\begin{array}{r}
\operatorname{div}(K \nabla q)=\operatorname{div}(K g) \quad \text { in } \quad \Omega, \\
K \nabla q \cdot n=K g \cdot n \quad \text { on } \quad \partial \Omega,
\end{array}
$$

where $K$ is the symmetric and positive tensor defined by

$$
K_{j i}=\int_{Y} w_{j}^{i} .
$$

Taking into account that the homogenized problem (5.7)-(5.8) is wellposed, then by straightforward verification we obtain the complete asymptotic behaviour of the solution:

Theorem 8 If $\left(u^{\varepsilon}, p^{\varepsilon}\right) \in H_{\varepsilon} \times L_{0}^{2}(\Omega)$ is the solution of (3.19)-(3.20), then

$$
\begin{gathered}
u^{\varepsilon} \stackrel{2}{\rightarrow} u=\left(g_{i}-\frac{\partial q}{\partial x_{i}}\right) w^{i}, \\
p^{\varepsilon} \stackrel{2}{\rightarrow} p=q,
\end{gathered}
$$

where $w^{i} \in W$ and $q \in \tilde{H}^{1}(\Omega)$ are defined by (5.11) and (5.13)-(5.14). 
Acknowledgements. This work has been accomplished during the visit of D. Polişevschi at the I.R.M.A.R.'s Department of Mechanics (University of Rennes 1), whose support is gratefully acknowledged.

\section{References}

1. Allaire, G: Homogenization of the Stokes flow in a connected porous medium, Asymptotic Analysis 2, 203-222 (1989).

2. Allaire, G: Homogenization and two-scale convergence, S.I.A.M. J. Math. Anal. 23 1482-151 (1992).

3. Barenblatt, G.I., Zheltov, Y.P., Kochina, I.N.: On basic conceptions of the theory of homogeneous fluids seepage in fractured rocks (in Russian), Prikl.Mat. i Mekh. 24, 852864 (1960).

4. Barenblatt, G.I., Entov, V.M., Ryzhik, V.M.: Theory of Fluid Flows Through Natural Rocks, Kluwer Acad. Pub., Dordrecht (1990).

5. Beavers, G.S., Joseph, D.D.: Boundary conditions at a naturally permeable wall, J. Fluid Mech. 30, 197-207 (1967).

6. Cioranescu, D., Saint Jean-Paulin, J.: Homogenization in open sets with holes, J. Math. Anal. Appl. 71 (2), 590-607 (1979).

7. Conca, C.: On the application of the homogenization theory to a class of problems arising in fluid mechanics, J. Math. Pures et Appl. 64, 31-75 (1985).

8. Ene, H.I., Poliševski, D.: Model of diffusion in partially fissured media, Z.A.M.P. 53 1052-1059 (2002).

9. Girault, V., Raviart, P.-A.: Finite Element Methods for Navier-Stokes Equations, Springer-Verlag, Berlin (1986).

10. Gruais , I., Polisevski, D.: Fluid flows through fractured porous media along BeaversJoseph interfaces, To appear in J. Math. Pures et Appl..

11. Jäger, W., Mikelić, A.: Modeling effective interface laws for transport phenomena between an unconfined fluid and a porous medium using homogenization, Transp. Porous Med. 78, 489-508 (2009).

12. Jones, I.P.: Low Reynolds number flow past a porous spherical shell, Proc Camb. Phil. Soc. 73, 231-238 (1973).

13. Lipton, R., Avellaneda, M.: Darcy's law for slow viscous flow past a stationary array of bubbles, Proc. Roy. Soc. Edinburgh Sect. A 114 (1-2), 71-79 (1990).

14. Mikelić, A.: Effets inertiels pour un écoulement stationnaire visqueux incompressible dans un milieu poreux, C. R. Acad. Sci. Paris Sér. I Math.320 (10), 1289-1294 (1995).

15. Neuss-Radu, M.: Some extensions of two-scale convergence. C.R. Acad. Sci. Paris 322, Série I, 899-904 (1996).

16. Nguetseng, G.: A general convergence result for a functional related to the theory of homogenization, S.I.A.M. J.Math.Anal. 20, 608-623 (1989).

17. Poliševski, D.: Basic homogenization results for a biconnected $\epsilon$-periodic structure, Appl. Anal. 82(4), 301-309 (2003).

18. Saffman, P.G.: On the boundary condition at the interface of a porous medium. Stud. Appl. Math. 50, 93-101 (1971).

19. Sanchez-Palencia, E.: Non-Homogeneous Media and Vibration Theory. Lecture Notes in Physics, vol. 127, Springer-Verlag, Berlin (1980).

20. Tartar, L.: Incompressible fluid flow in a porous medium. Convergence of the homogenization process. Appendix of [19]. 